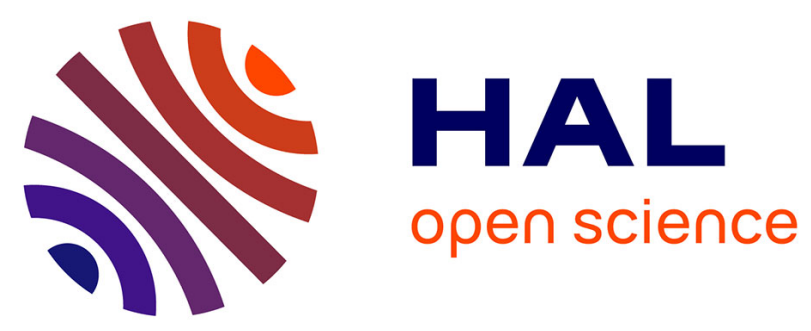

\title{
Double buffer circuit for the characterization of piezoelectric nanogenerators based on $\mathrm{ZnO}$ nanowires
}

Kevin Nadaud, François Morini, Abhishek Singh Dahiya, Camille Justeau, Sarah Boubenia, Kiron P Rajeev, Daniel Alquier, Guylaine Poulin-Vittrant

\section{- To cite this version:}

Kevin Nadaud, François Morini, Abhishek Singh Dahiya, Camille Justeau, Sarah Boubenia, et al.. Double buffer circuit for the characterization of piezoelectric nanogenerators based on $\mathrm{ZnO}$ nanowires. Applied Physics Letters, 2018, 112 (6), pp.063901. 10.1063/1.5018145 . hal-01727273

\section{HAL Id: hal-01727273 \\ https://hal.science/hal-01727273}

Submitted on 9 Mar 2018

HAL is a multi-disciplinary open access archive for the deposit and dissemination of scientific research documents, whether they are published or not. The documents may come from teaching and research institutions in France or abroad, or from public or private research centers.
L'archive ouverte pluridisciplinaire HAL, est destinée au dépôt et à la diffusion de documents scientifiques de niveau recherche, publiés ou non, émanant des établissements d'enseignement et de recherche français ou étrangers, des laboratoires publics ou privés. 


\section{Double buffer circuit for the characterization of piezoelectric nanogenerators based on} ZnO nanowires

Kevin Nadaud, ${ }^{1}$ a) François Morini, ${ }^{1}$ Abhishek S. Dahiya, ${ }^{1}$ Camille Justeau, ${ }^{1}$ Sarah

Boubenia, ${ }^{1}$ Kiron P. Rajeev, ${ }^{1}$ Daniel Alquier,${ }^{1}$ and Guylaine Poulin-Vittrant ${ }^{1}$

GREMAN UMR 7347, Université de Tours, CNRS, INSA-CVL,

16 rue Pierre et Marie Curie, 37071 Tours, France

The accurate and precise measurements of voltage and current output generated by a nanogenerator (NG) is crucial to design the rectifying/harvesting circuit and to evaluate correctly the amount of energy provided by a NG. High internal impedance of the NG's (several $M \Omega$ ) is the main limiting factor for designing circuits to measure the open circuit voltage. In this paper, we present the influence of the characterization circuit used to measure the generated voltage of piezoelectric NGs. The proposed circuit consists of a differential amplifier which permits to measure the voltage provided by the NG without applying any parasitic bias to it. The proposed circuit is compared to a commercial electrometer and a homemade buffer circuit based on a voltage follower circuit to show its interest. For the proposed double buffer circuit, no asymmetric behavior has been noticed contrary to the measurements made using simple buffer circuit and Keithley electrometer. The proposed double buffer circuit is thus suitable to measure the NG voltage in a transparent way, as an ideal voltage probe should do.

Keywords: energy harvesting, nano-generators, piezoelectrics, $\mathrm{ZnO}$ nanowires, electrical characterization

a)Electronic mail: kevin.nadaud@univ-tours.fr 
The recent growth of the Internet of Things (IoT) has led to an increasing demand for the development of low power energy sources. Compact, miniature and low power batteries offers a potential solution for the realization of the IoT. However their implementation are restricted due to the finite amount of energy stored in them. In order to overcome such limitations, various technologies of harvesters have been investigated for mechanical energy harvesting since the early 2000's. In the last decade, a new generation of piezoelectric harvesters have been developed, based on piezoelectric semiconducting nanowires, called nanogenerators (NGs) ${ }^{1-5}$. NGs can be used to provide the electrical energy to devices such as sensors, actuators and wireless transmitters ${ }^{6-9}$ The advantage of the piezoelectric NGs is their ability to collect the otherwise wasted mechanical energy, such as human body movements and ambient vibrations. NGs can be used to power an electronic device directly using a capacitor ${ }^{10,11}$ and also can be placed in tandem to charge a small embedded battery ${ }^{12,13}$. With a view to reduce the usage of lead, nanomaterials such as $\mathrm{ZnO}$ nanowires have been investigated in NG's due their piezoelectric properties and bio-compatibility ${ }^{1-3}$.

Careful characterization of such NGs is crucial in order to accurately determine their performances and to precisely quantify the harvested power. For example, when measuring the generated voltage, the measured voltage $V_{m}$ is lower than the open circuit voltage $V_{O C}$ due to the internal impedance of the measuring equipment (Fig. 1): ${ }^{14-16}$

$$
V_{m}=\frac{Z_{L}}{Z_{L}+Z_{N G}} V_{O C}
$$

with $Z_{L}$ the internal impedance of the measurement system and $Z_{N G}$ the internal impedance of the NG. Thus, to measure the open circuit voltage, it is necessary to connect the NG to a very large impedance (ideally higher than $100 \mathrm{M} \Omega$ ) compared to its internal impedance. Nevertheless, conventional oscilloscopes present an internal impedance around $1 \mathrm{M} \Omega$ which is not sufficient to measure $V_{O C}$ and this loading has a strong effect on the measured voltage.

In order to overcome the loading problem, it is possible to use a high impedance probe whose internal impedance is usually $100 \mathrm{M} \Omega$. The main drawback of this approach is the division of the measured voltage by a factor 100 and thus the reduction in the accuracy of the measured values. A solution is to use a voltage follower circuit made with an operational amplifier (op-amp) as shown in Fig. 2, called the "simple buffer circuit". Theoretically, a voltage follower has an infinite input impedance but, in practice, the input impedance is around $1 \mathrm{~T} \Omega$ for FET-input op-amp such as OPA604 of TL081. Such high impedance values are enough to measure voltages at open-circuit condition. 


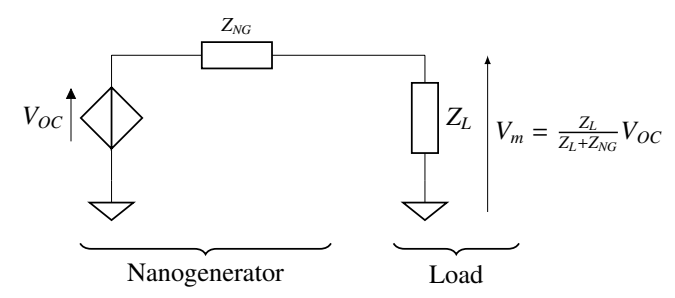

Figure 1. Illustration of the loading effect during the measurement of the nanogenerators.

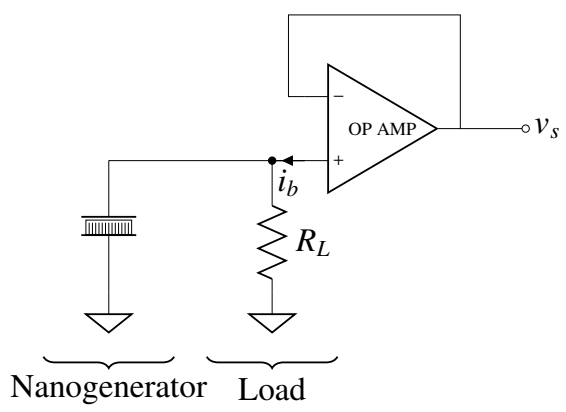

Figure 2. Schematic of the simple buffer circuit based on voltage follower for the measurement of the nanogenerator voltage.

Usually, during the characterization of an energy converter, such as piezoelectric NGs or components having a capacitive behavior, a load resistance $\left(R_{L}\right)$ is necessary to provide a return path to the ground for the biasing current of the op-amp. It means, even if the goal is to measure the open circuit voltage, that it is not possible to connect the NG directly to the non-inverting input without an additional resistor acting as a return path to the ground. As a current is flowing into this resistance $R_{L}$, a potential difference appears across it. The higher the resistance $R_{L}$, the higher the bias voltage, so a compromise should be found between a large $R_{L}$ value (compared to the NG internal impedance, in order to limit the loading effect) and a low $R_{L}$ value (in order to limit the bias voltage). It is also important to choose an op-amp with a very low value of the biasing current (for example, a FET-input op-amp) to limit the magnitude of the bias voltage. Nevertheless, when the bias voltage is applied to the $\mathrm{NG}$ and can change its behavior ${ }^{17,18}$. The use of a high impedance electrometer, such as 6517A Keithley electrometer in voltage mode, is possible for the characterization of the NG. Nevertheless, this electrometer also has a follower circuit at the first stage and thus needs a load resistance for the return path to the ground.

In this paper, we propose the study of a differential amplifier, called double buffer circuit, which permits to reduce the bias voltage effect during the measurement of the voltage generated by the 


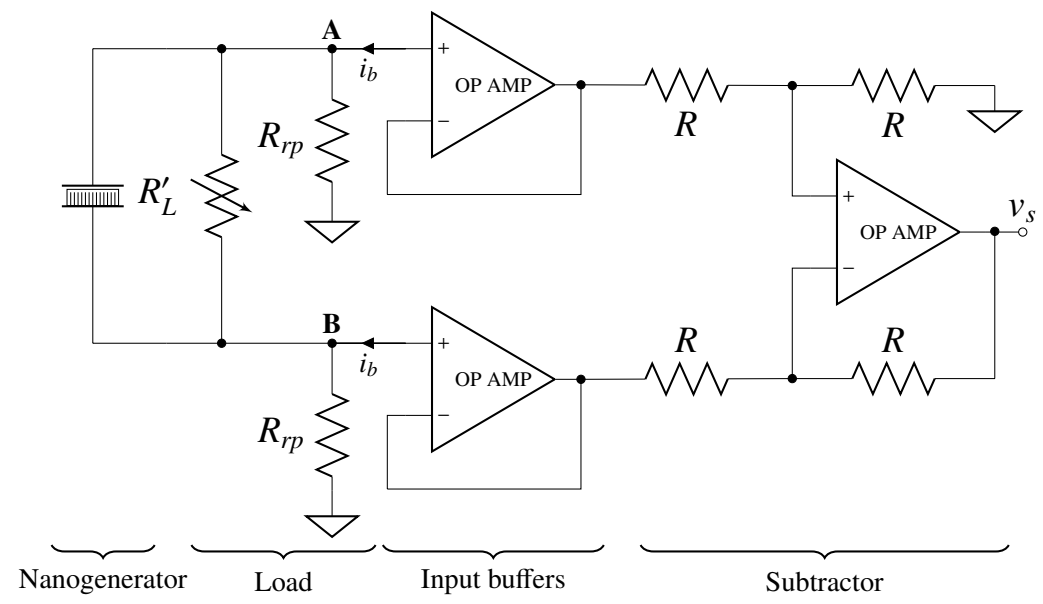

(a)

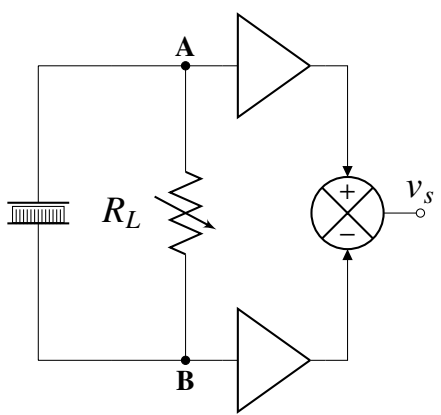

(b)

Figure 3. Schematic of the proposed double buffer circuit. Details of the implementation (a) and simplified view (b).

NG. The performances of the double buffer circuit are compared to those of both the simple buffer circuit and the Keithley electrometer 6517A.

The piezoelectric NGs studied in this paper are based on $\mathrm{ZnO}$ nanowires. A low-temperature hydrothermal method is chosen for the growth of $\mathrm{ZnO}$ nanowires, described elsewhere ${ }^{19}$. The nanowires are grown on a polydimethylsiloxane (PDMS) substrate coated with Ti/Au (100 nm/ $200 \mathrm{~nm}$ ) films deposited using e-beam evaporation ${ }^{20}$. A $\mathrm{ZnO}$ nucleation layer deposited by sputtering is used for the $\mathrm{ZnO}$ nanowire growth. The nanowires are then encapsulated into a parylene-C polymer matrix. $\mathrm{Ti} / \mathrm{Al}(100 \mathrm{~nm} / 400 \mathrm{~nm})$ layers are then deposited by e-beam evaporation to realize the top contact electrode. Electrical contacts are achieved by soldering copper wires onto the top (Al) and bottom (Au) electrodes using silver epoxy paste. Finally the device is encapsulated in a polydimethylsiloxane (PDMS) film. The $1 \times 1 \mathrm{~cm}^{2}$ active area is subjected to a low frequency compressive force using a home-made test bench setup ${ }^{2}$. The test-bench includes a mechanical shaker (LDS V406) equipped with a ridged aluminium actuator arm, a power amplifier (LDS PA100E) used to drive the shaker and a function generator (Agilent 33 250) used to control the magnitude and frequency of the shaker ${ }^{2}$. The magnitude and frequency of the force used are $3 \mathrm{~N}$ and $5 \mathrm{~Hz}$ respectively. The electrical characterization is performed using three different circuits: (i) a Keithley 6517A electrometer in voltage mode, (ii) the simple buffer circuit as described Fig. 2 and (iii) the proposed double buffer circuit which will be described later. The output of the circuits are connected to an Agilent DSO5054A oscilloscope for the acquisition of the voltage responses. 
To compensate the bias effect, a double buffer circuit is proposed, as presented in Fig. 3(a) with its equivalent simplified view shown in Fig. 3(b). It consists of 2 voltage followers connected to a subtractor stage. It is very similar to a conventional instrumentation amplifier structure, commonly available in Integrated Circuits (IC). The major difference is the gain which is fixed to 1 since the goal is to measure the NG signal and not to amplify it. The double buffer circuit is similar to the differential charge amplifier presented in ref. 21 , however, in our case, the considered signal is voltage not charges. The principle is to have both electrical terminals of the NG at the same potential, a condition which is fulfilled by connecting them to two identical voltage followers. To extract the desired signal, a subtractor stage is used. The TL084 has been chosen because of its very high input impedance (1 T $\Omega$ typically) and its very low input bias current (30 pA typically), in order to limit the effect of the bias voltage. Moreover, since all the op-amps are from the same IC, the characteristics are very close to each other, which permits to reduce the current difference and thus the bias voltage difference between the two nodes of the NG. The load resistance $R_{L}^{\prime}$ is connected between the two follower inputs. In order to provide a return path for the bias current of each buffer, the $R_{r p}$ resistances are connected to the ground and a value of $1 \mathrm{G} \Omega$ is chosen in order to reduce their influence on the load of the NG. The effective loading resistance of the NG is thus:

$$
R_{L}=\frac{2 R_{L}^{\prime} R_{r p}}{R_{L}^{\prime}+2 R_{r p}}
$$

In our work, all the measurements are performed at an effective load resistance of $100 \mathrm{M} \Omega$. The effective load resistance value was achieved in the double buffer circuit by choosing a resistor $R_{L}^{\prime}=105 \mathrm{M} \Omega$ in order to take into account the return path resistors $R_{r p}$ whose value are $1 \mathrm{G} \Omega$. With the aim of a complete study of the NG, the load resistance $R_{L}^{\prime}$ can be swept in order to find the optimal load which maximizes the output power ${ }^{10}$.

The fabricated NG has been characterized using both the Keithley electrometer and the simple buffer circuit. The results are shown in Fig. 4. We clearly observed a variation in the electrical response depending on the electrodes connection, as shown in Fig. 4. Whereas for the forward connection (top electrode connected to the input of the measurement circuit and bottom electrode to the ground), the peak to peak voltage is $1.8 \mathrm{~V}$. For the reverse connection, (top electrode connected to the ground and bottom electrode to input of the measurement circuit), the peak to peak voltage is $64 \mathrm{mV}$ which is significantly lower value compared with the one observed for the forward connection. The asymmetric behavior has been already noticed in ref. 17 and 18. In our case, the generated voltage is much higher. Time waveforms measured using the simple buffer circuit 


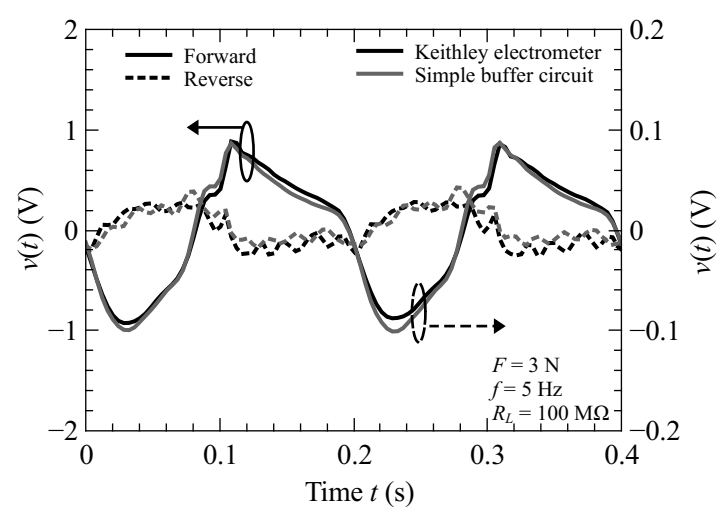

Figure 4. NG measured voltage using Keithley electrometer and simple buffer circuit as a function of the time for forward en reverse connections of the NG.

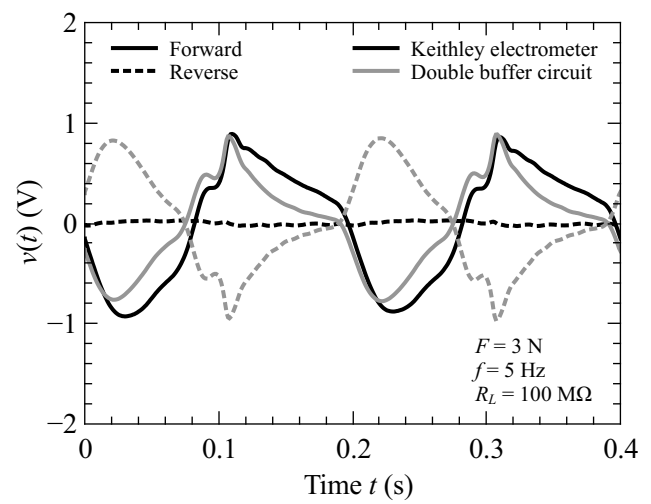

Figure 5. NG measured voltage using Keithley electrometer and double buffer circuit as a function of the time for forward en reverse connections of the NG.

are very similar to those of the Keithley electrometer, which suggests that the asymmetric behavior may come from the topology of the buffer and not from a design problem of the home-made simple buffer circuit.

Fig. 5 shows the measured voltage using the double buffer circuit compared to the Keithley electrometer. In the case of the double buffer circuit, forward connection refers to the top electrode connected to the non-inverting input of circuit (A) and the bottom electrode connected to the inverting input (B). Reverse connection refers to the reciprocal case (bottom electrode connected to input $\mathbf{A}$ and top electrode connected to input $\mathbf{B}$ ). Time waveforms measured using the double buffer are very symmetric, which shows the reversibility of the voltage measurement principle.

To confirm that the asymmetric behavior comes from the bias of the buffers, different configurations have been tested with the double buffer circuit and are presented in Fig. 6. For all those 


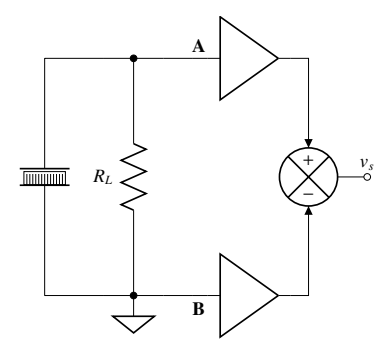

(a)

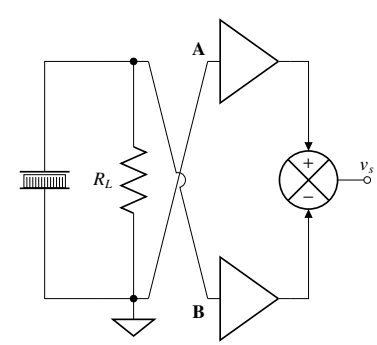

(c)

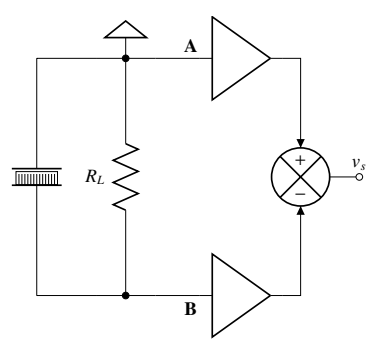

(b)

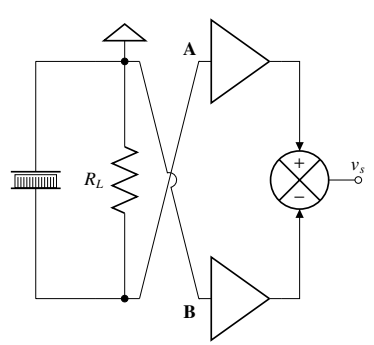

(d)

Figure 6. Connections of the different studied cases. (a) NG top electrode on double buffer non-inverting input, bottom electrode grounded. (b) NG top electrode grounded, bottom electrode on double buffer inverting input. (c) NG top electrode on double buffer inverting input, bottom electrode grounded. (d) NG top electrode grounded, bottom electrode on double buffer non-inverting input.

configurations, a $R_{L}^{\prime}$ value of $110 \mathrm{M} \Omega$ has been chosen in order to keep an effective loading of $100 \mathrm{M} \Omega$ since one of the return path resistor $R_{r p}$ is shortened. Fig. 6(a) and Fig. 6(c) are similar in the sense that the bottom electrode is connected to the ground. The only difference comes from the input on which the top electrode is connected to: the non-inverting input for 6(a) and the inverting input for 6(c). In these cases, since both buffers are identical, the bias applied to the NG top electrode is identical. Configurations, presented in Fig. 6(b) and Fig. 6(d), are similar in the sense that the top electrode is connected to the ground. The difference comes from the input to which the bottom electrode is connected, to the non-inverting input for 6(d) and to the inverting input for 6(b). The output voltage vs time characteristics of $\mathrm{NG}$ in various connection scenarios are shown in Fig. 7.

From the Fig. 7, it is observed that when the top electrode is connected to the ground, the generated signal is very low which corresponds well to the reverse connection scenario with both the simple buffer circuit and the Keithley electrometer (shown in Fig. 5). When the bottom electrode is connected to the ground, and thus the top electrode connected to one input of the buffer, the generated voltage is higher and similar to what is obtained in the forward connection. The difference 


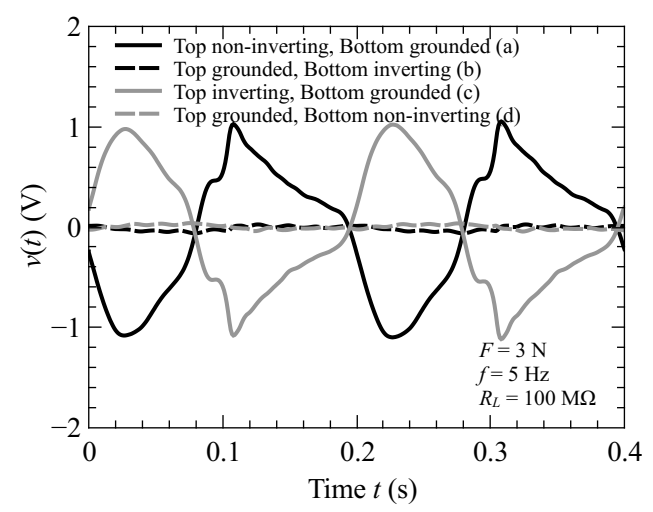

Figure 7. Measured NG voltage signals for the different cases described Fig. 6.

of polarity comes from the chosen input of the circuit (inverting or non inverting).

The proposed circuit can be used in order to determine the optimal load impedance which maximizes the output power. To do so, the resistance between inputs $R_{L}^{\prime}$ can be varied (see Fig. 3(a)) and the effective loading of the NG can be calculated using (2). Since both inputs stay symmetrical when the load resistance changes, the proposed circuit still operates as an ideal probe.

The generated voltage has been measured while changing the load resistance for both connections (forward and reverse). Since the load is resistive, the output power can be obtained using the following formula:

$$
P_{\text {average }}=\frac{\left(V_{R M S}\right)^{2}}{R_{L}}
$$

with $V_{R M S}$ the root mean square voltage:

$$
V_{R M S}=\sqrt{\frac{1}{T} \int_{0}^{T}\left(v_{s}(t)\right)^{2} \mathrm{~d} t}
$$

In our case, the RMS value has been calculated over 5 periods.

Fig. 8 shows the measured generated RMS voltage and average power as functions of the load resistance $R_{L}$, using the double buffer circuit. The measured voltage and computed power are the same for both connections showing that the variation of the resistance does not change the working principle of the double buffer circuit. In our case, the optimal load may be slightly higher than $100 \mathrm{M} \Omega$ since the maximum of power is not reached.

This paper highlights the importance of the method employed to measure the voltage delivered by a piezoelectric nanogenerator dedicated to mechanical energy harvesting. It is critical to choose the proper voltage measurement circuit so that the voltage response is not disturbed by the current bias of the op-amp included in the measurement circuit. Whatever the chosen measurement circuit, 


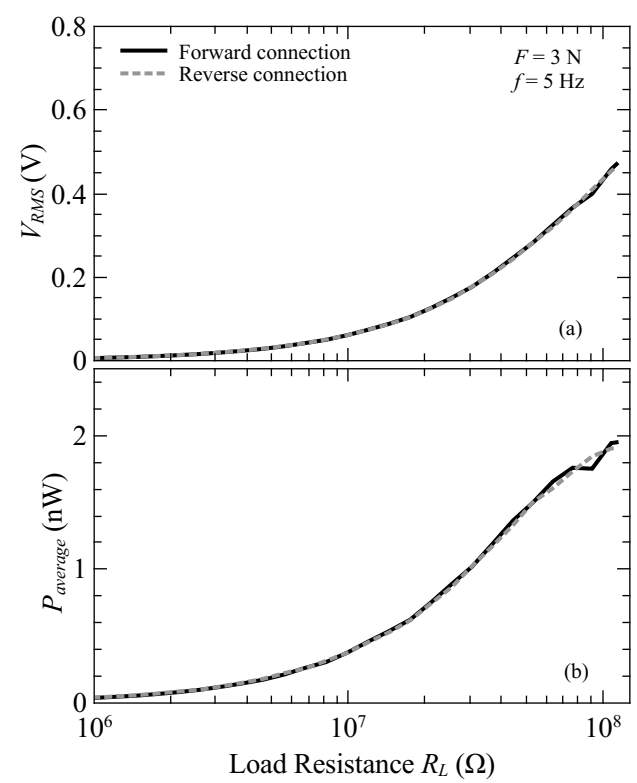

Figure 8. Measured RMS voltage generated by the NG and its average power as a function of the load resistance $R_{L}$.

the NG voltage magnitude may depend on the way the NG electrodes are connected to the input stage of the measurement circuit. This influence is attributed to the bias current circulating at the input of the buffer. Thus, in this work, we show that a double buffer circuit permits to measure the NG voltage in a transparent way, as an ideal voltage probe should do. This amplifier is in such case more symmetric than a conventional simple buffer circuit and thus has no impact on the nanogenerator. With the proposed double buffer circuit, the NG voltage amplitude remains unchanged, when inverting the NG electrodes connected to the double buffer. In addition, the proposed double buffer still operates when changing the resistance between inputs, in order to find the optimal load which maximizes the output power. This work demonstrates the importance to use the appropriate voltage measurement circuit in order to get reliable results when performing the functional characterization of piezoelectric nanogenerators.

See supplementary material for additional informations about the topology of the studied nanogenerator and for SEM image of the nanowires.

This project has received funding from the ECSEL JU under grant agreement $\mathrm{N}^{\circ} 692482$. This JU receives support from the European Union's H2020 research and innovation programme and France, Netherlands, Denmark, Belgium, Germany, Czek Republic, Spain. The Authors are also grateful for the supports from Region Centre (MEPS project 2015-2018) and National Research 
Agency (ANR-14-CE08-0010-01).

\section{REFERENCES}

${ }^{1}$ G. Zhu, A. C. Wang, Y. Liu, Y. Zhou, and Z. L. Wang, Nano Letters 12, 3086 (2012).

${ }^{2}$ C. Opoku, A. S. Dahiya, F. Cayrel, G. Poulin-Vittrant, D. Alquier, and N. Camara, RSC Adv. 5, 69925 (2015).

${ }^{3}$ R. Tao, M. Parmar, G. Ardila, P. Oliveira, D. Marques, L. Montès, and M. Mouis, Semiconductor Science and Technology 32, 064003 (2017).

${ }^{4}$ N. Gogneau, N. Jamond, P. Chrétien, F. Houzé, E. Lefeuvre, and M. Tchernycheva, Semiconductor Science and Technology 31, 103002 (2016).

${ }^{5}$ S. Xu, B. J. Hansen, and Z. L. Wang, Nature Communications , 93 (2010).

${ }^{6}$ Y. Hu, Y. Zhang, C. Xu, L. Lin, R. L. Snyder, and Z. L. Wang, Nano Letters 11, 2572 (2011).

${ }^{7}$ S. Roundy and P. K. Wright, Smart Materials and Structures 13, 1131 (2004).

${ }^{8}$ Z. Lin, J. Chen, X. Li, Z. Zhou, K. Meng, W. Wei, J. Yang, and Z. L. Wang, ACS Nano 11, 8830 (2017).

${ }^{9}$ S. Xu, Y. Qin, C. Xu, Y. Wei, R. Yang, and Z. L. Wang, Nature Nanotechnology 5, 366 (2010).

${ }^{10}$ M. Marzencki, Y. Ammar, and S. Basrour, Sensors and Actuators A: Physical 145, 363 (2008).

${ }^{11}$ P. Miribel-Català, J. Colomer-Fararons, J. L. Brinquis, and J. López-Sánchez, in Design of Circuits and Integrated Systems (2014) pp. 1-6.

${ }^{12}$ D. Zhu, S. P. Beeby, M. J. Tudor, and N. R. Harris, Sensors and Actuators A: Physical 169, 317 (2011).

${ }^{13}$ H. Wei, D. Cui, J. Ma, L. Chu, X. Zhao, H. Song, H. Liu, T. Liu, N. Wang, and Z. Guo, J. Mater. Chem. A 5, 1873 (2017).

${ }^{14}$ Y. Su, C. Dagdeviren, and R. Li, Advanced Functional Materials 25, 5320 (2015).

${ }^{15}$ J. Briscoe, N. Jalali, P. Woolliams, M. Stewart, P. M. Weaver, M. Cain, and S. Dunn, Energy Environ. Sci. 6, 3035 (2013).

${ }^{16}$ J. Dicken, P. D. Mitcheson, I. Stoianov, and E. M. Yeatman, IEEE Transactions on Power Electronics 27, 4514 (2012).

${ }^{17}$ R. Yang, Y. Qin, C. Li, L. Dai, and Z. L. Wang, Applied Physics Letters 94, 022905 (2009).

${ }^{18}$ Z. Li, G. Zhu, R. Yang, A. C. Wang, and Z. L. Wang, Advanced Materials 22, 2534 (2010).

${ }^{19}$ S. Boubenia, A. S. Dahiya, G. Poulin-Vittrant, F. Morini, K. Nadaud, and D. Alquier, Scientific 
Reports 7, 15187 (2017).

${ }^{20}$ A. S. Dahiya, F. Morini, S. Boubenia, K. Nadaud, D. Alquier, and G. Poulin-Vittrant, Advanced Materials Technologies (2017), 10.1002/admt.201700249, 1700249.

${ }^{21}$ V. F. Rivera, F. Auras, P. Motto, S. Stassi, G. Canavese, E. Celasco, T. Bein, B. Onida, and V. Cauda, Chemistry - A European Journal 19, 14665 (2013). 